\title{
Custom contact lenses and cornea-scleral contact lenses from irregular astigmatism
}

\begin{abstract}
Objective: To show the improvement of visual acuity in five clinical cases of irregular astigmatism, achieved with contact lenses of different materials and geometries.

Case reports: Five case reports are exposed and their adaptations for the use of this type of lenses are evaluated. After inserting corneo-scleral lenses or soft contact lenses, each adaptation is evaluated.

Results: All patients have improved meaningfully their visual acuity without affecting their corneal integrity and improving their quality of life.

Conclusion: Advances in the design and materials of contact lenses have helped eyecare professionals to solve visual problems that would have been difficult to solve years ago.
\end{abstract}

Keywords: irregular astigmatism, keratoconus, keratoplasty, custom contact lenses,

cornea-scleral contact lenses
Volume 10 Issue 2 - 2020

\author{
Pérez Silguero D, Encinas Pisa P, Bermal \\ Blasco I, Pérez Silguero MA \\ Health clinics Pérez Silguero, Spain
}

Correspondence: Encinas Pisa P, Health clinics Pérez Silguero, Spain, calle leon y castillo 58,35003, Tel 686578699.

Email pablo.encinas.pisa@gmail.com

Received: April 09, 2020 | Published: April 16, 2020

\section{Introduction}

The cornea is the first eye tissue that light must pass through on its way to the retina. The refractive power of the same is about 43 diopters (D) with the total power of the eye approximately $60 \mathrm{D}$. Its shape is prolate, aspherical and regular, all necessary to achieve quality vision. In order to compensate for the excess or deficiency of the cornea's power, we can correct it with refractive surgeries or compensate it with ophthalmic lenses or contact lenses (LC). ${ }^{1}$ In this work, 5 clinical cases of patients suffering from some type of refractive problem secondary to irregular astigmatisms are presented, which have been compensated with different LC materials and geometries. It is worth noting the importance of a good diagnosis to be able to choose the most appropriate LC for each patient in order to provide comfort and greater visual acuity (VA) while respecting corneal integrity at all times.

\section{Cynical cases}

\section{MFC case}

Female patient attended in our service since 2006, when we diagnosed a bilateral grade III keratoconus in RE and grade II in LE (Amsler-Krumeich classification), with a VA of 0.3 and 0.5 respectively. (Woman who comes to our consultation since 2006, at 10 years of age and a greater bilateral keratoconus is detected in OD than in OI); positive Munson's sign in both eyes (Figure 1). Until the end of 2015, a total of 3 crosslinking was performed in each eye. During that time, the patient wore soft molded contact lenses that did not produce good VA, in addition to showing low tolerance.

RX OD:-10 spheres -2.00 cyl at $15^{\circ}=\mathrm{AV} 0.8$ difficult

RX OI: -5.75 sph -3.75 cyl at $138^{\circ}=$ AV 0.7. We adapt Markennovy custom designed soft contact lenses (LC); Saphir $\mathrm{Rx}^{\circledR}$.

The final lenses were:

OD:-9.00-1.5 cyl at $15^{\circ}=\mathrm{AV} 1.0 \mathrm{Rb}: 8.0$ Diameter: 15 .
OI:-5.75-3.75 cyl at $138^{\circ}=\mathrm{AV} 1.0 \mathrm{Rb}: 8.0$ Diameter: 15 .

We used the 3 D OCT 2000 from the Topcon house, giving postcontact tear meniscus measurements of 110 microns in OD and 142 in OI (Figure 2). None of the radial cuts that OCT allows us to observe a corneal touch. As a result, we have a correct adaptation that allows our patient to enjoy better VA to function in their daily life.

\section{ERG case}

A 28-year-old male underwent penetrating keratoplasty in 2015 with an $8.5 \mathrm{~mm}$ donor button in LE, due to herpes in 2013.

RX OI-5.00 cyl at $100^{\circ}=\mathrm{AV} 0.05$.

We adapt Markennovy custom designed soft LCs; Saphir Rx ${ }^{\circledR}$.

The final lens was:

RX:-4.75 cyl at $100^{\circ}=\mathrm{AV} 0.5 \mathrm{Rb}: 8.3$ Diameter: 15 .

We used the 3 D OCT 2000 from the Topcon house giving measurements of the 305 micron post-contact tear meniscus in LE (Figure 3). Corneal touch is not observed in any of the radial cuts that our OCT allows us.

\section{EPR case}

A 31-year-old man with bilateral III degree keratoconus in each eye. In 2011, two corneal segments were inserted in the LE and in 2015 a segment in the OD. It has an AV SC in OD of 0.6 and 0.1 in OI.

RX OD:-7.75 sph-3.00 cyl 70 AC AV 0.6.

RX OI-16.50 sph-8.00 cyl 168 ; AV 0.2.

We adapt Tiedra corneo-scleral LCs; Alexa ES ${ }^{\circledR}$.

The final lens was:

OD RB 6.7 Diameter 15 LAZ 4 Power-15 AV 0.8.

OI RB 6.7 Diameter 15 LAZ 5 Power-14 AV 0.8 diff. 
It should be noted that in this adaptation several geometry changes were made due to the indentation produced due to corneal irregularity; VA of 1.0 in OD and 0.9 in OI were also achieved, which monocularly was well tolerated by the user but binocularly had problems with image duplication and worsening of VA at close range. The postlent meniscus was 300 microns in OD (Figure 4) and 200 microns in LE with absence of touch of the lens and completely saving the inserted segments.

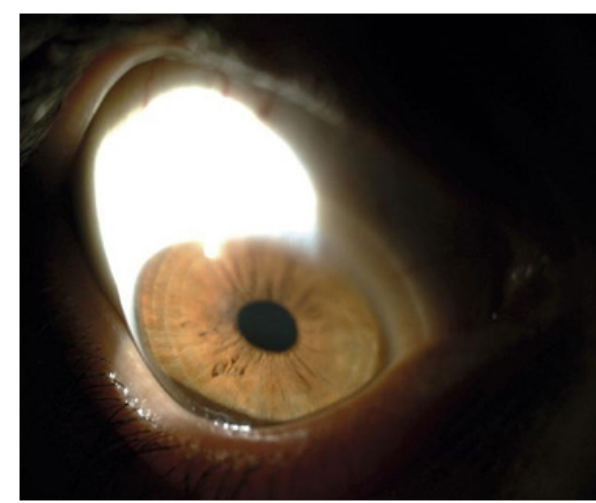

Figure I Munson's sign.

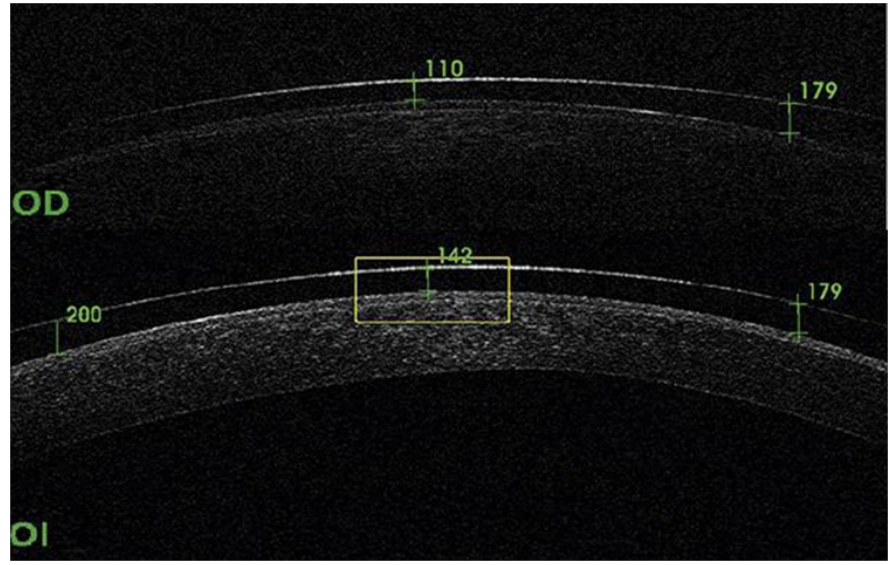

Figure $2 \mathrm{OCT}$ anterior $\mathrm{AO}$.

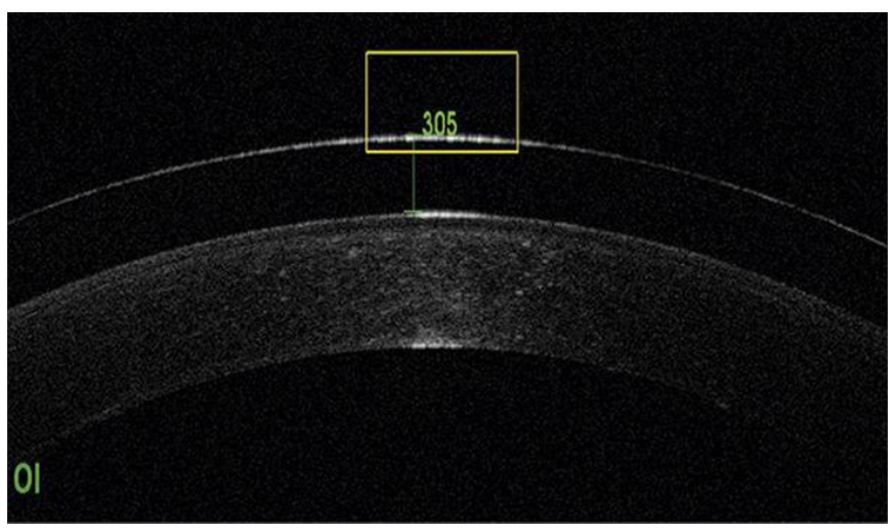

Figure $3 \mathrm{OCT}$ anterior $\mathrm{AO}$.

\section{APJ case}

45-year-old male operated on with bilateral keratoconus and operated on for penetrating keratoplasty in LE in 2004. After surgery, the patient suffers from Urrets Zavalia syndrome. In 2006, he underwent surgery with two corneal segments in OD.

RX OD:-15.75 sph-7.75 cyl at $150^{\circ} \mathrm{AV} 0.05$.

RX OI-12.25 spf-7.25 at $61^{\circ} \mathrm{AV}<0.05$.

We decided to adapt a Tiedra corneo-scleral LC; Alexa ES ${ }^{\circledR}$ in OD.

The final lens was:

OD RB 6.50 Diameter 15 LAZ 2 Power-10.50 AV 1.0.

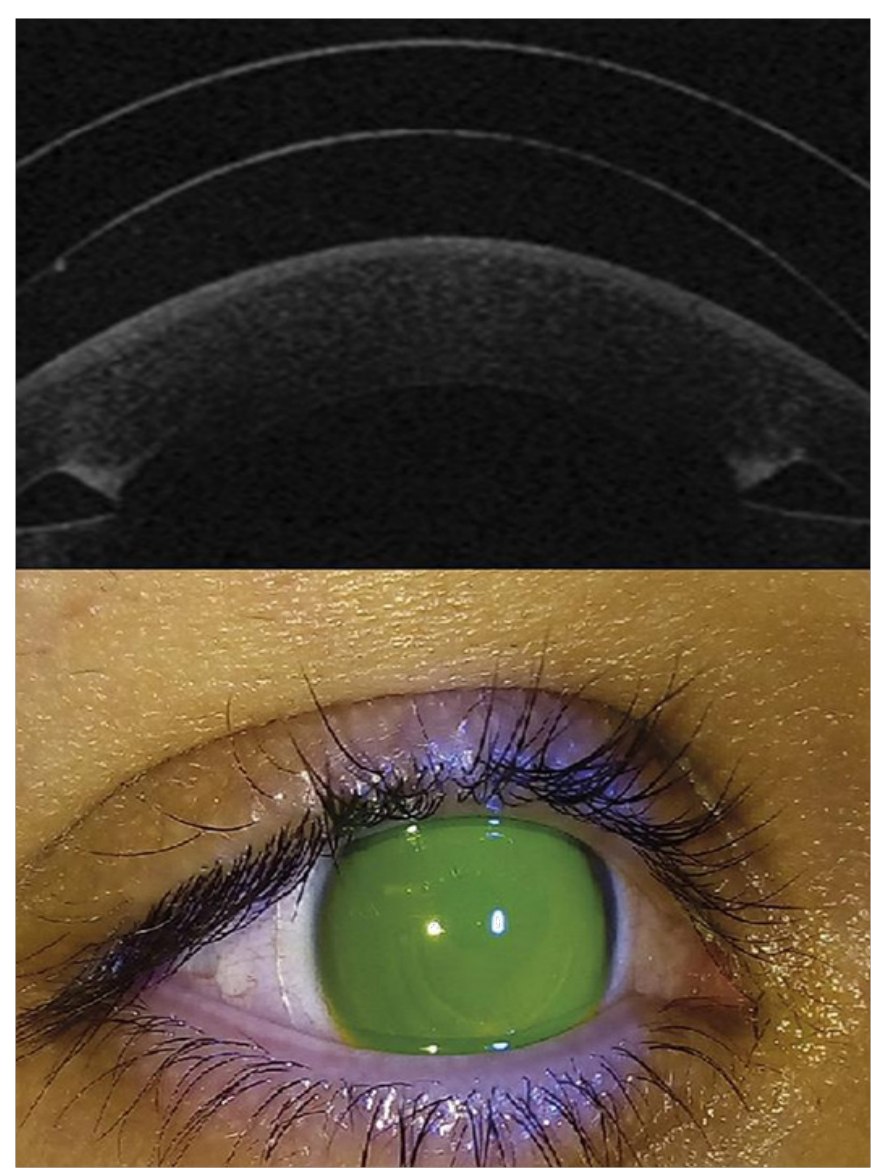

Figure $4 \mathrm{OCT}$ anterior $\mathrm{OD}$.

The postlent meniscus was 358 microns in OD as soon as the lens was inserted (Figure 5) and 306 microns in 5 hours of wear. The lens has no touch of the lens and totally saves the inserted segments. It should be noted that he had been on sick leave since keratoplasty and has currently been able to join his job by improving VA.

\section{CLC case}

73-year-old female patient underwent keratoplasty in $\mathrm{OC}$ and subsequent phacoemulsification.

He has a binocular VA $<0.05$.

We decided to adapt a Tiedra corneo-scleral LC; Alexa ES ${ }^{\circledR}$ in OD.

The final lens was:

OD RB 7.20 Diameter 15 LAZ 6 Power-5.00 AV 0.9 (Figure 6).

We used the 3 D OCT 2000 of the Topcon house giving measurements of 321 micron post-contact tear meniscus in OI. Corneal 
touch is not observed in any of the radial cuts that our OCT allows us. The closest approach was found in the lower temporal zone and was 60 microns.

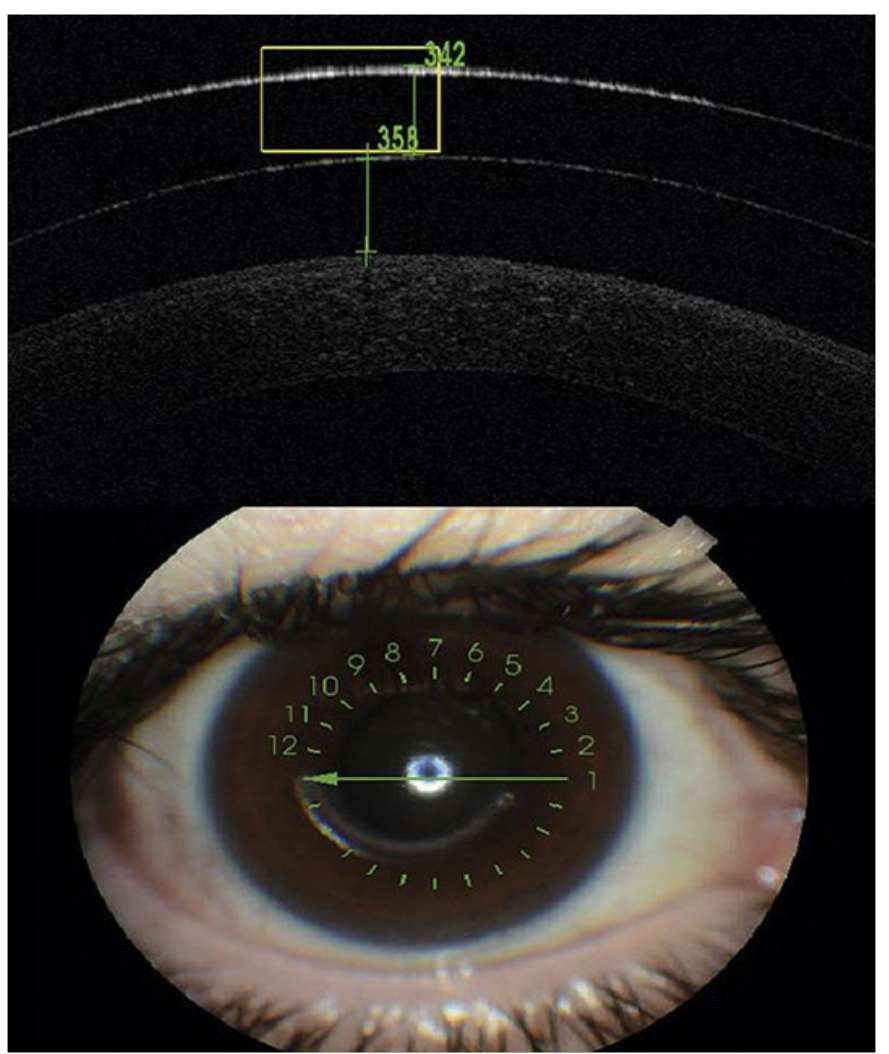

Figure 5 OCT anterior OD.

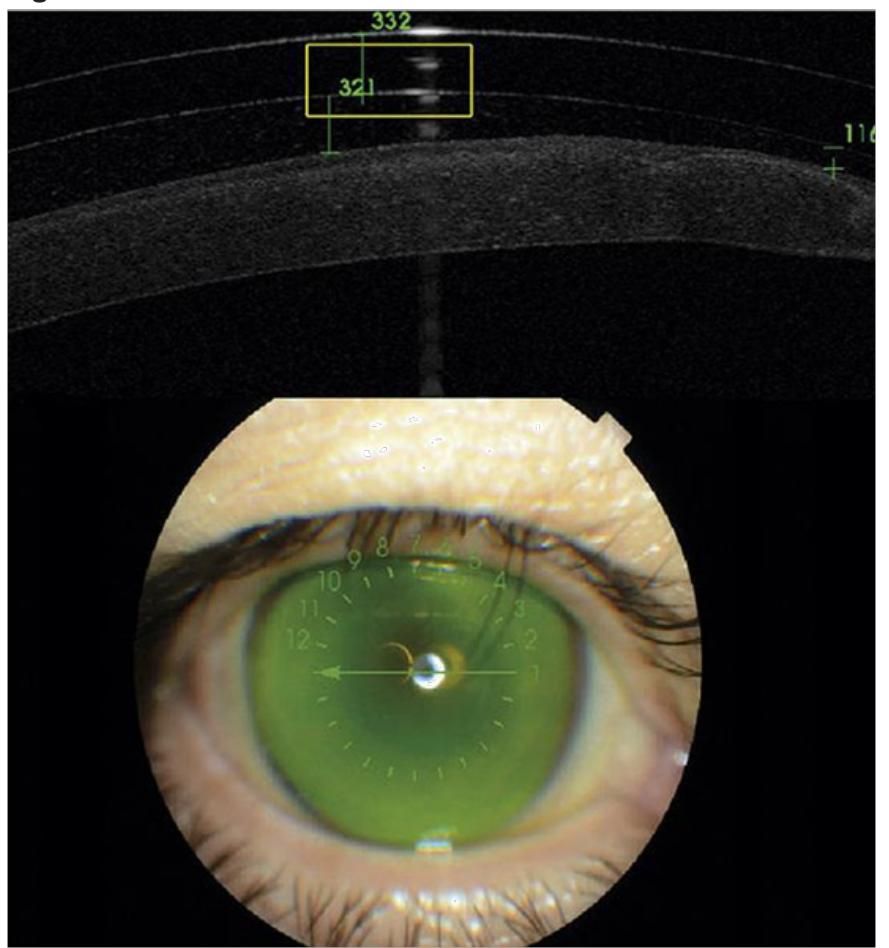

Figure 6 OD final lens.

\section{Discussion}

Currently, there are many manufacturers and contact lens geometries on the market that make it possible to improve the quality of life for this type of patient. There are cases of mild irregular astigmatism in which a good VA can be achieved with a soft, molded lens, but higher-grade irregular astigmatisms require the use of special contact lens types; Whether they are soft, custom designed and turned manufacturing, or rigid corneal, corneo-scleral, or scleral contact lenses that allow us to regularize the corneal surface through the postcontact meniscus. If we have the appropriate manufacturing range, we could try adapting the soft contact lens, ${ }^{2}$ but keep in mind that in moderate irregular astigmatisms to try to make the post-contact meniscus simulate some corneal regularity, the lens should have a high stiffness modulus (Mpa). In cases of keratoplasty or keratoconus with high irregular astigmatisms, the most correct option is a rigid lens as in the case of corneo-scleral lenses ${ }^{3,4}$ since they support the sclera, avoiding any type of friction in the corneal area and creating a postlente lacrimal reservoir between 100 and 300 microns. The support area or edge of these lenses can also be regulated in many cases to avoid indentation of the same in the sclera with the risks of bleaching of blood vessels that this entails.

\section{Conclusion}

Advances in contact lens designs and materials, both in rigid gas permeable and in soft contact lenses, have made it possible for us to solve visual problems of patients that years ago would have been difficult to solve, such as irregular astigmatisms, keratoplasties or corneal ectasias. It is necessary to increase the research and development of this type of lens to improve the quality of life of these patients.

\section{Acknowledgments \\ None.}

\section{Funding}

None.

\section{Conflicts of interest}

Author declares that there is no conflict of interest.

\section{References}

1. Rico-Del-Viejo L, Garcia-Montero M, Hernández-Verdejo JL, et al. Nonsurgical Procedures for Keratoconus Management. $J$ Ophthalmol. 2017;9707650.

2. Chen YW, Lee JS, Hou CH, et al. Correction of hyperopia with astigmatism following radial keratotomy with daily disposable plus spherical contact lens: a case report. Int Ophthalmol. 2018;38(5):2199-2204.

3. Baali M, Belghmaidi S, Ahammou H, et al. Evaluation of the quality of life of patients fitted with scleral lenses using a Moroccan version of NEI-VFQ 25. J Fr Ophtalmol. 2018;41(3):201-205.

4. Porcar E, Montalt JC, Spain-Gregori E, et al. Corneo-scleral contact lenses in an uncommon case of keratoconus with high hyperopia and astigmatism. Cont Lens Anterior Eye. 2017;40(5):351-356. 